\title{
Natural interpretation on the data of $\Lambda_{c} \rightarrow \Sigma \pi$
}

\author{
Hong-Wei Ke $\odot^{1, *}$ and Xue-Qian $\mathrm{Li}^{2, \dagger}$ \\ ${ }^{1}$ School of Science, Tianjin University, Tianjin 300072, China \\ ${ }^{2}$ School of Physics, Nankai University, Tianjin 300071, China
}

(Received 3 September 2020; accepted 1 December 2020; published 24 December 2020)

\begin{abstract}
Even though the Standard Model has achieved great success, its application to the field of low energy still lacks solid foundation due to our limited knowledge on nonperturbative QCD. Practically, all theoretical calculations of the hadronic transition matrix elements are based on various phenomenological models. There indeed exist some anomalies in the field that are waiting for interpretations. The goal of this work is trying to solve one of the anomalies: the discrepancy between the theoretical prediction on the sign of the up-down asymmetry parameter of $\Lambda_{c} \rightarrow \Sigma \pi$ and the experimental measurement. In the literatures several authors calculated the rate and determined the asymmetry parameter within various schemes, but there exist obvious loopholes in those adopted scenarios. To solve the discrepancy between theory and data, we suggest that not only the direct transition process contributes to the observed $\Lambda_{c} \rightarrow \Sigma \pi$, but also other portals such as $\Lambda_{c} \rightarrow \Lambda \rho$ play a substantial role via an isospin-conserving rescattering $\Lambda \rho \rightarrow \Sigma \pi$. Taking into account the effects induced by the final state interaction, we reevaluate the relevant quantities. Our theoretical prediction based on this scenario involving an interference between the direct transition of $\Lambda_{c} \rightarrow \Sigma \pi$ and the portal $\Lambda_{c} \rightarrow \Lambda \rho \rightarrow \Sigma \pi$ can make both the decay rate and sign of the asymmetry parameter to be consistent with data.
\end{abstract}

DOI: $10.1103 /$ PhysRevD.102.113013

\section{INTRODUCTION}

Even though the Standard Model (SM) has achieved great success, its application to the field of low energy still lacks solid foundation due to our limited knowledge on nonperturbative QCD. Practically, all theoretical calculations of the hadronic transition matrix elements are based on various phenomenological models. There indeed exist some anomalies in the field that are waiting for interpretations. One of the anomalies is the discrepancy between the theoretical prediction on the sign of the up-down asymmetry parameter of $\Lambda_{c} \rightarrow \Sigma \pi$ and the experimental measurement. In fact, besides the meson case, for baryons that contain three ingredients, their complexity makes a thorough study on them more difficult than on mesons. However from another aspect, the involved physics in the transitions between baryons is also richer and by the research one can get better understanding of the governing mechanisms. An advantage of studying decays of baryons

\footnotetext{
khw020056@tju.edu.cn

†lixq@nankai.edu.cn
}

Published by the American Physical Society under the terms of the Creative Commons Attribution 4.0 International license. Further distribution of this work must maintain attribution to the author(s) and the published article's title, journal citation, and DOI. Funded by SCOAP ${ }^{3}$. involving heavy flavors is obvious just as one does on the heavy mesons (structure, production, and decay).

Especially, the charmed hadrons are of special significance because charm quark is heavier than the light quarks $(u, d, s)$, but at the same time is not as heavy as the bottom quark, so that relativistic effects are not negligible at all. The issue that the lifetimes of $B^{ \pm}, B^{0}$, and $\Lambda_{b}$ are close, however, the lifetimes of $D^{ \pm}, D^{0}$, and $\Lambda_{c}$ are quite apart, has been warmly discussed. It is believed that the Pauli interference induces the lifetime difference between $D^{ \pm}$ and $D^{0}[1,2]$, which is suppressed for the $B$ hadrons, but for $\Lambda_{c}$ the question still exists.

Since 1990s, many decay channels of $\Lambda_{c}$ have successively been measured by experimental collaborations [3-6], and the field has attracted attentions of theorists. Its weak decays have been carefully explored with different approaches [7-15]. In this work, we would revisit the old topic because much larger datasets with higher precision are available at BESIII, Belle, and even LHCb, which make us hope to get a better understanding on the charmed baryons.

Among the previous theoretical studies on the decay rate of $\Lambda_{c} \rightarrow \Sigma \pi$ and the corresponding up-down asymmetry parameter $\alpha$, the pole model was adopted because of its advantage. The pole model is simple and the relevant parameters are adopted by fitting data, therefore one can trust its effectiveness. A naïve conjecture would expect that the prediction obtained with this model should be 
TABLE I. Decay width $\Gamma$ (in units of $\times 10^{-14}$ ) and up-down asymmetry $\alpha$ of $\Lambda_{c} \rightarrow \Sigma \pi$.

\begin{tabular}{lccccccrr}
\hline \hline & Ref. [7] & Ref. [8] & Ref. [9] & Ref. [10] & Ref. [11] & Ref. [12] & Ref. [13] & \multicolumn{1}{c}{ Exp. [22] } \\
\hline$\Gamma$ & 1.10 & 3.03 & 1.34 & 1.17 & 2.48 & 6.07 & 4.62 & $4.08 \pm 0.33$ \\
$\alpha$ & 0.70 & 0.43 & 0.39 & 0.92 & 0.83 & -0.49 & -0.31 & $-0.45 \pm 0.32$ \\
\hline \hline
\end{tabular}

consistent with data even though an error is unavoidable in this relatively rough picture. However, it is noticed that the prediction on the up-down asymmetry parameter $\alpha$ is positive while the measured value is negative. This apparent discrepancy which is not a tolerable deviation, indicates that there must be something wrong. Thus to solve this "anomaly" there are two routes. One is that the method adopted for the calculation should be modified whereas another possibility is that besides the direct transition, there exist other contributions to the observed data on $\Lambda_{c} \rightarrow \Sigma \pi$. As the first route, Cheng and his collaborators [12] went on to use the current algebra calculating the transition matrix element $\left\langle\Sigma\left|H_{\text {eff }}\right| \Lambda_{c}\right\rangle$ and obtained a negative $\alpha$. However, the precondition of using the current algebra [16] is properly extracting the pion field out from the matrix element $\left\langle\pi \Sigma\left|H_{\text {eff }}^{\prime}\right| \Lambda_{c}\right\rangle$ under the soft-pion approximation. By contrast for the process $\Lambda_{c} \rightarrow \Sigma \pi$, the three momentum of the pion is not small to be "soft"; thus the whole scenario is questionable.

Instead, we follow the second route; i.e., the obvious loophole may suggest that there could be another mechanism. We propose that other channels of $\Lambda_{c}$ decays would contribute to the observed $\Lambda_{c} \rightarrow \Sigma \pi$ via final state interactions. The interference between the direct transition and the new contribution may lead to the results consistent with data. Considering the effective interaction and the decay rate of $\Lambda_{c}$ decays, the most possible two-step process is that $\Lambda_{c}$ first transits into $\Lambda \rho$ then, by a rescattering, $\Lambda \rho$ turns into $\Sigma \pi$ and its contribution would interfere with the amplitude of direct transition $\Lambda_{c} \rightarrow \Sigma \pi$.

As is well known, the weak decays of heavy hadrons mainly occur via an emission of virtual $W$ or $Z$ bosons which later turn into lepton or quark pairs, from the heavy quark (antiquark). In our case, $\Lambda_{c}$ is an isospin singlet, therefore the $u$ - $d$ subsystem (one may call it as a diquark) in $\Lambda_{c}$ exists in an isospin singlet $(I=0)$, and it is noted that the $u$ - $d$ subsystem in $\Lambda$ is also an isospin singlet, but in $\Sigma^{0}$ the subsystem is an isospin-triplet $(I=1)$. During the transition of $\Lambda_{c} \rightarrow \Lambda$ the $u$ - $d$ subsystem serves generally as a spectator and retains its isospin unchanged, whereas for $\Lambda_{c} \rightarrow \Sigma^{0}$, the isospin of the $u-d$ subsystem is forced to change from a singlet into a triplet. Practically, for the $\Lambda_{c} \rightarrow \Sigma^{0} \pi^{+}$transition, the $W$ boson emitted by the charm quark must be connected to the $u-d$ subsystem, which is no longer a real spectator. Although the weak interaction does not conserve isospin, the isospin analysis may help us to get an insight into what happens during the transition.
According to the analysis given in literature [11], the rate of $\Lambda_{c} \rightarrow \Lambda \rho$ should be about twice larger than that of $\Lambda_{c} \rightarrow \Sigma \pi$, therefore a two-step process, $\Lambda_{c} \rightarrow \Lambda \rho \rightarrow \Sigma \pi$, may substantiate and change the picture (especially the sign of the asymmetry parameter). This is not a surprise to notice the role of final interaction: in our earlier work, we studied the case of $D^{0} \rightarrow K^{0} \bar{K}^{0}$, which has the same rate as $D^{0} \rightarrow K^{+} K^{-}$. In fact the former is strongly suppressed, nevertheless the later is favored. The result is fully understood as $D^{0} \rightarrow K^{0} \bar{K}^{0}$ is realized via a rescattering $K^{+} K^{-} \rightarrow$ $K^{0} \bar{K}^{0}$ [17]. The rescattering mechanism also has been successfully applied to explain some other anomalies existing in low energy experiments, such as the decays of $\Upsilon$ and bottomed mesons [18-21], thus we have a full confidence that the mechanism also works well here.

Therefore in this work we include the contributions from both the direct transition and that induced by the final state interaction, and their interference leads to the final result which is experimentally measured. For a comparison, in the following Table I, we list the results given in literature. Concretely, all the coupled channels of $\Lambda_{c}$ should contribute to the observed $\Lambda_{c} \rightarrow \Sigma \pi$ via final state interactions (rescattering). In terms of the effective interaction, the corresponding coupling constants and the first step of the sequential $\Lambda_{c}$ decays, the process $\Lambda_{c} \rightarrow \Lambda \rho \rightarrow \Sigma \pi$ should be dominant one.

This paper is organized as follows: after this introduction we will consider the contribution to $\Lambda_{c} \rightarrow \Sigma \pi$ by including the final state interactions. In Sec. III we present our numerical results. Section IV is devoted to a brief summary.

\section{THE SEQUENTIAL DECAY $\Lambda_{c} \rightarrow \Lambda \rho \rightarrow \Sigma \pi$}

The amplitude of baryon decays $\mathcal{B}_{i} \rightarrow \mathcal{B}_{f} P$ can be written as [11]

$$
\mathcal{M}\left(\mathcal{B}_{i} \rightarrow \mathcal{B}_{f} P\right)=i \bar{U}_{\mathcal{B}_{f}}\left[A-B \gamma_{5}\right] U_{\mathcal{B}_{i}}
$$

where $\mathcal{B}_{i}\left(\mathcal{B}_{f}\right)$ is the initial (final) baryon and $P$ is a pseudoscalar meson.

For the transition $\mathcal{B}_{i} \rightarrow \mathcal{B}_{f} V$, the amplitude is

$$
\begin{aligned}
\mathcal{M}\left(\mathcal{B}_{i} \rightarrow \mathcal{B}_{f} V\right)= & i \bar{U}_{\mathcal{B}_{f}} \varepsilon^{* \mu}\left[-A_{1} \gamma_{\mu} \gamma_{5}-A_{2} p_{f \mu} \gamma_{5}\right. \\
& \left.+B_{1} \gamma_{\mu}+B_{2} p_{f \mu}\right] U_{\mathcal{B}_{i}},
\end{aligned}
$$

where $V$ is a vector meson with polarization $\varepsilon$ and $p_{f}$ is the momentum of $\mathcal{B}_{f}$. It is noted that the sign of the $\gamma_{5}$ term in 
Ref. [11] is opposite to the convention adopted in [15]. Here with this minus sign in front of every item involving $\gamma_{5}$ (conventional definition), all the values of $B, A_{1}$, and $A_{2}$ given in Ref. [11] do not need to be changed and we directly adopt their formulas. Indeed. this provides us a great convenience to derive relevant quantities.

For the decay $\Lambda_{c} \rightarrow \Sigma \pi$ the factors $A$ and $B$ should include the contributions of all relevant Feynman diagrams. At the quark level the transition does not occur via factorizable Feynman diagrams but those of nonfactorizable ones [7,11]. In Ref. [11] the authors employed the simple pole-model to calculate the contributions of nonfactorizable Feynman diagrams. However the sign of the up-down asymmetry $\alpha$ gained in this way is opposite to data.

It is easy to conjecture that in this case, the contribution from the rescattering of final state in some decays of $\Lambda_{c}$ might play an important role. The goal of this work is just to check if the rescattering of the final products can change the scenario, namely simultaneously producing results in the required production rate for $\Lambda_{c} \rightarrow \Sigma \pi$ and a correct updown asymmetry $\alpha$. In principle many coupled channels would jointly contribute to the decay $\Lambda_{c} \rightarrow \Sigma \pi$, for example $\Lambda_{c} \rightarrow \Lambda \rho$ and $\Lambda_{c} \rightarrow \Lambda \pi$ etc. Considering the coupling constants and the rates of the first step decay of $\Lambda_{c}$ one can decide that the main process is $\Lambda_{c} \rightarrow \Lambda \rho \rightarrow \Sigma \pi$, where the second step is the isospin conserving rescattering $\Lambda \rho \rightarrow \Sigma \pi$.

The total amplitude of the practical transition $\Lambda_{c} \rightarrow \Sigma \pi$ is

$$
\begin{aligned}
M & =M^{\mathrm{DIR}}+M^{\mathrm{FSI}}, \\
& =i \bar{U}_{\Sigma}\left[A^{\mathrm{DIR}}-B^{\mathrm{DIR}} \gamma_{5}\right] U_{\Lambda_{c}}+i \bar{U}_{\Sigma}\left[A^{\mathrm{FSI}}-B^{\mathrm{FSI}} \gamma_{5}\right] U_{\Lambda_{c}}, \\
& =i \bar{U}_{\Sigma}\left[A-B \gamma_{5}\right] U_{\Lambda_{c}},
\end{aligned}
$$

where $M^{\mathrm{DIR}}$ and $M^{\mathrm{FSI}}$ correspond to the contributions of the direct transition $\Lambda_{c} \rightarrow \Sigma \pi$ and the two-step process $\Lambda_{c} \rightarrow \Lambda \rho^{+} \rightarrow \Sigma \pi^{+}$, respectively, $A=A^{\mathrm{DIR}}+A^{\mathrm{FSI}}$ and $B=B^{\mathrm{DIR}}+B^{\mathrm{FSI}}$. The amplitude of the direct transition $M^{\text {DIR }}$ was calculated in terms of the pole model [11] and we will use their numerical results directly.

Now let us begin to study the processes whose corresponding Feynmen diagrams are depicted in Fig. 1. We add subscribe $a(b)$ to the amplitudes of $\Lambda \rightarrow \Sigma^{0} \pi^{+}\left(\Lambda \rightarrow \Sigma^{+} \pi^{0}\right)$, i.e., $M_{a}, M_{a}^{\mathrm{DIR}}$, and $M_{a}^{\mathrm{FSI}}\left(M_{b}, M_{b}^{\mathrm{DIR}}\right.$, and $\left.M_{b}^{\mathrm{FSI}}\right)$.

Generally, the absorptive part overwhelmingly dominates and the contribution of the dispersive one can be ignored, so that we only need to calculate the absorptive part of the Feynmen diagrams in Fig. 1. where the intermediate states $\rho$ and $\Lambda$ are on shell and the transition amplitude of $\Lambda_{c} \rightarrow \Lambda \rho$ can also be written in terms of the pole model. The relevant effective interactions are [23]

$$
\begin{aligned}
\mathcal{L}_{\Lambda \Sigma \pi} & =g_{\Lambda \Sigma \pi} \bar{\psi}_{\Sigma} \gamma_{5} \psi_{\Lambda} \pi, \\
\mathcal{L}_{\rho \pi \pi} & =g_{\rho \pi \pi}\left(\partial_{\mu} \pi^{0} \pi^{+} \rho^{-\mu}-\partial_{\mu} \pi^{+} \pi^{0} \rho^{-\mu}\right) .
\end{aligned}
$$

It is noted that here for calculating the absorptive part of the triangle, we adopt the pseudoscalar form for the coupling $\mathcal{L}_{\Lambda \Sigma \pi}$. In principle there also exists the axial-vector form [24,25] for the $\Lambda \Sigma \pi$ coupling. However, in our case, $\Lambda$ and $\Sigma$ are on their mass shells, thus with help of the Dirac equation, one can easily show that the pseudoscalar coupling and the axial-vector one are equivalent, even though their coupling constants are different. We just take the proper one that is phenomenologically fixed by fitting corresponding data. By the Cutkosky rule, one can factorize the transition into two parts as $M(\Lambda \rightarrow \Lambda \rho) \times$ $M(\Lambda \rho \rightarrow \Sigma \pi)$. Let us first calculate the amplitude corresponding to diagram Fig. 1(a):

$$
\begin{aligned}
M_{a}^{\mathrm{FSI}}= & \frac{1}{2} \int \frac{d \mathbf{p}_{1}}{(2 \pi)^{3} 2 E_{1}} \frac{d \mathbf{p}_{2}}{(2 \pi)^{3} 2 E_{2}}(2 \pi)^{4} \delta\left(p-p_{1}-p_{2}\right) \mathcal{M}^{\mathrm{DIR}}\left[\Lambda_{c} \rightarrow \Lambda \rho\right] \mathcal{M}[\Lambda \rho \rightarrow \Sigma \pi], \\
= & \frac{1}{2} \int \frac{d \mathbf{p}_{1}}{(2 \pi)^{3} 2 E_{1}} \frac{d \mathbf{p}_{2}}{(2 \pi)^{3} 2 E_{2}}(2 \pi)^{4} \delta\left(p-p_{1}-p_{2}\right) i \bar{U}_{\Lambda}\left[-A_{1}^{\mathrm{DIR}} \gamma_{\mu} \gamma_{5}-A_{2}^{\mathrm{DIR}} p_{1 \mu} \gamma_{5} \gamma_{\mu}\right. \\
& \left.+B_{1}^{\mathrm{DIR}}+B_{2}^{\mathrm{DIR}} p_{1 \mu}\right] U_{\Lambda_{c}} g_{\Sigma \Lambda \pi} \bar{U}_{\Sigma} \gamma_{5} U_{\Lambda} g_{\rho \pi \pi}(-i)\left(p_{4}+q\right)_{\nu}\left(-g^{\mu \nu}+\frac{p_{2}^{\mu} p_{2}^{\nu}}{m_{\rho}^{2}}\right) \frac{i}{q^{2}-m_{\pi}^{2}} F^{2}\left(q^{2}, m_{\pi}^{2}\right), \\
= & i \int \frac{\left|\mathbf{p}_{1}\right| d \Omega}{32 \pi^{2} E} \bar{U}_{\Lambda}\left[-A_{1}^{\mathrm{DIR}} \gamma_{\mu} \gamma_{5}-A_{2}^{\mathrm{DIR}} p_{1 \mu} \gamma_{5}+B_{1}^{\mathrm{DIR}} \gamma_{\mu}+B_{2}^{\mathrm{DIR}} p_{1 \mu}\right] U_{\Lambda_{c}} \bar{U}_{\Sigma} \gamma_{5} U_{\Lambda} \\
& \times g_{\Sigma \Lambda \pi} g_{\rho \pi \pi}\left(p_{4}+q\right)_{\nu}\left(-g^{\mu \nu}+\frac{p_{2}^{\mu} p_{2}^{\nu}}{m_{\rho}^{2}}\right) \frac{F^{2}\left(q^{2}, m_{\pi}^{2}\right)}{q^{2}-m_{\pi}^{2}}, \\
= & i \int \frac{\left|\mathbf{p}_{1}\right| d \Omega}{32 \pi^{2} E} \bar{U}_{\Sigma} \gamma_{5}\left(p_{1} A m_{\Lambda}\right)\left[-A_{1}^{\mathrm{DIR}} \gamma_{\mu} \gamma_{5}-A_{2}^{\mathrm{DIR}} p_{1 \mu} \gamma_{5}+B_{1}^{\mathrm{DIR}} \gamma_{\mu}+B_{2}^{\mathrm{DIR}} p_{1 \mu}\right] U_{\Lambda_{c}} \\
& \times g_{\Sigma \Lambda \pi} g_{\rho \pi \pi}\left(p_{4}+q\right)_{\nu}\left(-g^{\mu \nu}+\frac{p_{2}^{\mu} p_{2}^{\nu}}{m_{\rho}^{2}}\right) \frac{F^{2}\left(q^{2}, m_{\pi}^{2}\right)}{q^{2}-m_{\pi}^{2}},
\end{aligned}
$$




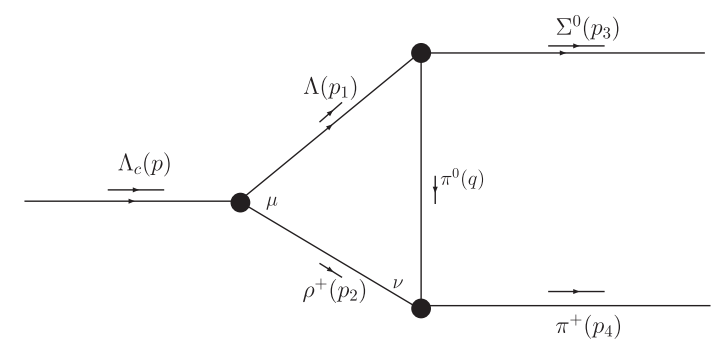

(a)

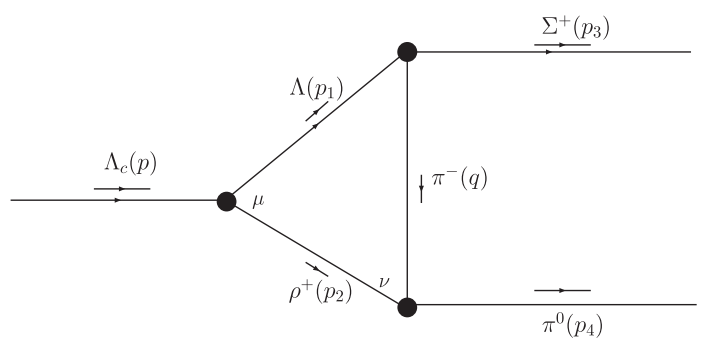

(b)

FIG. 1. The main final state interaction for $\Lambda_{c} \rightarrow \Sigma^{0} \pi^{+}$(a) and $\Lambda_{c} \rightarrow \Sigma^{+} \pi^{0}$ (b).

where $E, E_{1}$, and $E_{2}$ are the energies of $\Lambda_{c}, \Lambda$, and $\rho$, with $p$, $p_{1}, p_{2}, p_{3}$, and $p_{4}$ being the momenta of $\Lambda_{c}, \Lambda, \rho, \Sigma$, and $\pi, q$ is the momentum of the exchanged intermediate pions. Since in practice meson and baryon are not point particles, a form factor at each effective vertex should be introduced. The form factor suggested by many researchers is in the form:

$$
F\left(q^{2}, m_{P}^{2}\right)=\frac{\Lambda_{1}^{2}-m_{P}^{2}}{\Lambda_{1}^{2}-q^{2}},
$$

where $\Lambda_{1}$ is a cutoff parameter and $m_{P}$ is equal to $m_{\pi}$. Since the form factor is not derived from a fundamental principle, and the concerned cutoff parameter is neither determined theoretically, actually so far we know little about the cutoff parameter $\Lambda_{1}$. Generally $\Lambda_{1}$ is about $1 \mathrm{GeV}$ for the exchange of light mesons [19,21].

Using the four-momentum relations $p_{4}=p-p_{3}$, $q=p_{1}-p_{3}, p_{2}=p-p_{1}$ and contracting the indices $\mu$ and $\nu$, these notations $\not p, \not p_{1}, \not p_{3}, p^{2}, p_{1}^{2}, p_{3}^{2}, p \cdot p_{1}, p \cdot p_{3}$, and $p_{1} \cdot p_{3}$ appear in the expression of $M_{a}^{\mathrm{FSI}}$. One can employ Dirac equations $\not p U_{\Lambda_{c}}=m U_{\Lambda_{c}}$ and $\not p_{3} U_{\Sigma}=m_{3} U_{\Sigma}$ to simply the expression. Since $\Lambda_{c}, \Sigma$, and $\Lambda$ are on shell, $p^{2}, p_{1}^{2}, p_{3}^{2}, p \cdot p_{1}, p \cdot p_{3}$, and $p_{1} \cdot p_{3}$ can be expressed in terms of observable physical quantities. At last, one needs to deal with $\not p_{1}$. In our calculation we choose $\mathbf{p}_{3}$ in the $z$ direction, and the angle spanned between $\mathbf{p}_{1}$ and $\mathbf{p}_{3}$ is $\theta$. Since there exists an integration over azimuth one can find $\not \not_{1}=C_{1} \not \not+C_{2} \not \not_{3}$ with $C_{1}=\frac{E_{1}\left|\mathbf{p}_{3}\right|-\left|\mathbf{p}_{1}\right| E_{3} \cos \theta}{m\left|\mathbf{p}_{3}\right|}$ and $C_{2}=\frac{\left|\mathbf{p}_{1}\right| \cos \theta}{\left|\mathbf{p}_{3}\right|}$. Finally we obtain

$$
M_{a}^{\mathrm{FSI}}=i \bar{U}_{\Sigma}\left[A^{\mathrm{FSI}}-B^{\mathrm{FSI}} \gamma_{5}\right] U_{\Lambda_{c}},
$$

with $\quad A^{\mathrm{FSI}}=g_{\Sigma \Lambda \pi} g_{\rho \pi \pi} \int \frac{C_{a}\left|\mathbf{p}_{1}\right| \sin \theta F^{2}\left(q^{2}, m_{P}^{2}\right) d \theta}{16 \pi E} \quad$ and $\quad B^{\mathrm{FSI}}=$ $g_{\Sigma \Lambda \pi} g_{\rho \pi \pi} \int \frac{C_{b}\left|\mathbf{p}_{1}\right| \sin \theta F^{2}\left(q^{2}, m_{P}^{2}\right) d \theta}{16 \pi E}$. The detailed expressions of $C_{a}$ and $C_{b}$ are

$$
\begin{aligned}
& C_{a}=-A_{1}^{\mathrm{DIR}}\left[m^{3} m_{1}+m^{2} m_{1}{ }^{2}-m_{1}{ }^{4}-2 E_{3} m m_{1}\left(m+m_{1}\right)+m_{1}{ }^{2} m_{2}{ }^{2}+2 m_{1} m_{2}{ }^{2} m_{3}\right. \\
& \left.-m m_{1}\left(m_{1}^{2}+m_{2}^{2}-2 p_{1} \cdot p_{3}\right)+2 m_{1}^{2} p_{1} \cdot p_{3}-4 m_{2}^{2} p_{1} \cdot p_{3}\right] /\left[m_{2}^{2}\left(m_{\pi}^{2}-q^{2}\right)\right. \\
& -A_{2}^{\mathrm{DIR}} m_{1}\left[-2 E_{3} m m_{1}{ }^{2}+m^{2} m_{1}{ }^{2}-m_{1}{ }^{4}+m_{1}{ }^{2} m_{2}{ }^{2}+E_{1} m\left(2 E_{3} m-m^{2}+m_{1}{ }^{2}\right.\right. \\
& \left.\left.+m_{2}^{2}-2 p_{1} \cdot p_{3}\right)+2 m_{1}^{2} p_{1} \cdot p_{3}-2 m_{2}^{2} p_{1} \cdot p_{3}\right] /\left[m_{2}^{2}\left(m_{\pi}^{2}-q^{2}\right)\right] \\
& +\left(m C_{1}+m_{3} C_{2}\right) A_{1}^{\mathrm{DIR}}\left[-m^{3}-m^{2} m_{1}+m_{1}{ }^{3}+2 E_{3} m\left(m+m_{1}\right)-m_{1} m_{2}{ }^{2}+2 m_{2}{ }^{2} m_{3}\right. \\
& \left.+m\left(m_{1}^{2}+m_{2}^{2}-2 p_{1} \cdot p_{3}\right)-2 m_{1} p_{1} \cdot p_{3}\right] /\left[m_{2}^{2}\left(m_{\pi}^{2}-q^{2}\right)\right] \\
& -\left(m C_{1}+m_{3} C_{2}\right) A_{2}^{\mathrm{DIR}}\left[-2 E_{3} m m_{1}{ }^{2}+m^{2} m_{1}{ }^{2}-m_{1}{ }^{4}+m_{1}{ }^{2} m_{2}{ }^{2}+E_{1} m\left(2 E_{3} m\right.\right. \\
& \left.\left.-m^{2}+m_{1}^{2}+m_{2}^{2}-2 p_{1} \cdot p_{3}\right)+2 m_{1}^{2} p_{1} \cdot p_{3}-2 m_{2}^{2} p_{1} \cdot p_{3}\right] /\left[m_{2}^{2}\left(m_{\pi}^{2}-q^{2}\right)\right], \\
& C_{b}=B_{1}^{\mathrm{DIR}}\left[2 E_{3} m m_{1}\left(m-m_{1}\right)-m^{3} m_{1}+m^{2} m_{1}{ }^{2}+m m_{1}\left(m_{1}^{2}+m_{2}^{2}-2 p_{1} \cdot p_{3}\right)-m_{1}{ }^{4}\right. \\
& \left.+m_{1}^{2} m_{2}^{2}+2 m_{1}^{2} p_{1} \cdot p_{3}+2 m_{1} m_{2}^{2} m_{3}-4 m_{2}^{2} p_{1} \cdot p_{3}\right] /\left[m_{2}^{2}\left(m_{\pi}^{2}-q^{2}\right)\right] \\
& +B_{2}^{\mathrm{DIR}} m_{1}\left[E_{1} m\left(2 E_{3} m-m^{2}+m_{1}{ }^{2}+m_{2}{ }^{2}-2 p_{1} \cdot p_{3}\right)-2 E_{3} m m_{1}{ }^{2}+m^{2} m_{1}{ }^{2}-m_{1}{ }^{4}\right. \\
& \left.+m_{1}^{2} m_{2}^{2}+2 m_{1}^{2} p_{1} \cdot p_{3}-2 m_{2}^{2} p_{1} \cdot p_{3}\right] /\left[m_{2}^{2}\left(m_{\pi}^{2}-q^{2}\right)\right] \\
& +\left(m C_{1}-m_{3} C_{2}\right) B_{1}^{\mathrm{DIR}}\left[2 E_{3} m\left(m-m_{1}\right)-m^{3}+m^{2} m_{1}+m\left(m_{1}^{2}+m_{2}^{2}-2 p_{1} \cdot p_{3}\right)\right. \\
& \left.-m_{1}^{3}+m_{1} m_{2}^{2}+2 m_{1} p_{1} \cdot p_{3}-2 m_{2}^{2} m_{3}\right] /\left[m_{2}^{2}\left(m_{\pi}^{2}-q^{2}\right)\right] \\
& +\left(m C_{1}-m_{3} C_{2}\right) B_{2}^{\mathrm{DIR}}\left[E_{1} m\left(2 E_{3} m-m^{2}+m_{1}{ }^{2}+m_{2}{ }^{2}-2 p_{1} \cdot p_{3}\right)-2 E_{3} m m_{1}{ }^{2}+m^{2} m_{1}{ }^{2}\right. \\
& \left.-m_{1}{ }^{4}+m_{1}{ }^{2} m_{2}{ }^{2}+2 m_{1}{ }^{2} p_{1} \cdot p_{3}-2 m_{2}^{2} p_{1} \cdot p_{3}\right] /\left[m_{2}^{2}\left(m_{\pi}^{2}-q^{2}\right)\right],
\end{aligned}
$$


where $m, m_{1}, m_{2}, m_{3}$, and $m_{4}$ are the masses of $\Lambda_{c}, \Lambda, \rho, \Sigma$, and $\pi$, respectively, $E_{3}$ is the energy of $\Sigma$.

The decay rates of $\mathcal{B}_{i} \rightarrow \mathcal{B}_{f} P$ and up-down asymmetries are [11]

$\Gamma=\frac{\left|\mathbf{p}_{c}\right|}{8 \pi}\left[\frac{\left(m_{i}+m_{f}\right)^{2}-m_{P}^{2}}{m_{i}^{2}}|A|^{2}+\frac{\left(m_{i}-m_{f}\right)^{2}-m_{P}^{2}}{m_{i}^{2}}|B|^{2}\right]$,

$\alpha=\frac{2 \kappa \operatorname{Re}\left(A^{*} B\right)}{|A|^{2}+\kappa^{2}|B|^{2}}$,

where $\mathbf{p}_{c}$ is the three-momentum of $\mathcal{B}_{f}$ in the rest frame of $\mathcal{B}_{i}$ and $\kappa=\frac{\left|\mathbf{p}_{c}\right|}{E_{f}+m_{f}}$.

The amplitude of the direct transition $\Lambda_{c} \rightarrow \Sigma^{0} \pi^{+}$is straightforward calculated in terms of the pole model which was shown in the early works, so that we omit the details of the calculations in this section.

By the same process one can calculate the amplitudes of $\Lambda_{c} \rightarrow \Lambda \rho^{+} \rightarrow \Sigma^{+} \pi^{0}$, i.e., $M_{b}^{\mathrm{FSI}}$ [the absorptive part of the Feynman diagram, Fig. 1(b)]. We notice the sign of $M_{b}^{\mathrm{FSI}}$ is opposite to that of $M_{a}^{\mathrm{FSI}}$ but their magnitudes are same, which make the total amplitude $M_{b}$ is equal to $-M_{a}$ so the asymmetry parameters and decay widths of both $\Lambda_{c} \rightarrow$ $\Sigma^{+} \pi^{0}$ and $\Lambda_{c} \rightarrow \Sigma^{0} \pi^{+}$are same.

\section{THE THEORETICAL PREDICTIONS AS THE CONTRIBUTION FROM THE FINAL STATE INTERACTION IS TAKEN INTO ACCOUNT}

In a new work, the authors restudied the Cabibbosuppressed decays of $\Lambda_{c}$ [15] where the 30-year-old work $[11,12]$ was cited. It indicates, the fundamental framework does not change and therefore we can employ their numerical results directly. Moreover, $\Lambda_{c} \rightarrow \Lambda \rho$ has also been investigated [11], thus we just directly use their results about the first step transitions $M\left(\Lambda_{c} \rightarrow \Lambda \rho\right)$. Then we concentrate our attention on exploring the rescattering process $\Lambda \rho \rightarrow \Sigma \pi$ and analyze the consequences especially how inclusion of the final state interaction results in an

TABLE II. Theoretical results of $\Lambda_{c} \rightarrow \Sigma^{0} \pi^{+}$in pole model where $A^{\mathrm{fac}}, A^{\text {pole }}, B^{\mathrm{fac}}, B^{\text {pole }}$ are in units of $G_{F} V_{c s} V_{u d} \times$ $10^{-2} \mathrm{GeV}^{2}$ and $\Gamma$ is in units of $10^{-14} \mathrm{GeV}$.

\begin{tabular}{|c|c|c|c|c|c|c|}
\hline & $A^{\mathrm{fac}}$ & $A^{\text {pole }}$ & $B^{\mathrm{fac}}$ & $B^{\text {pole }}$ & $\alpha$ & $\Gamma$ \\
\hline$\overline{\Lambda_{c} \rightarrow \Sigma^{0} \pi^{+}}$ & 0 & 2.24 & 0 & 14.63 & 0.83 & 2.48 \\
\hline
\end{tabular}

opposite sign for the up-down asymmetry parameter from that determined by the direct transition.

We list the theoretical predictions given by the authors of Ref. [11] in Tables II and III for a clear reference. It is noted that the authors corrected their values about $\Lambda_{c} \rightarrow \Lambda \rho$ later and we employ the new ones for our numerical computations [line (a) in Table III]. Since the effective colorfavored Wilson coefficient $(\sim 1.315)$ used in Ref. [11] is larger than the present values $(\sim 1.26)$ [15], we set it to be 1.26 and repeated the calculations. The values we obtained are listed in line (b) of Table III.

In order to perform the numerical computations we need to determine the coupling constant $g_{\Sigma \Lambda \pi}$ and $g_{\rho \pi \pi}$. Using the data in particle data book [22] we fix $g_{\rho \pi \pi}=6.01$ and another factor $g_{\Sigma \Lambda \pi}=11.8$ was given in Ref. [11]. Generally the cutoff parameter $\Lambda_{1}$ is about $1 \mathrm{GeV}$ for a light exchanged meson. In our calculation we set it to be 0.8 and $1 \mathrm{GeV}$, respectively to make more sense. Using the formula derived above $A^{\mathrm{FSI}}$ and $B^{\mathrm{FSI}}$ are calculated with $A_{1}^{\mathrm{DIR}}=A_{1}^{\mathrm{fac}}+A_{1}^{\mathrm{pole}}, \quad A_{2}^{\mathrm{DIR}}=A_{2}^{\mathrm{fac}}+A_{2}^{\mathrm{pole}}, \quad B_{1}^{\mathrm{DIR}}=B_{1}^{\mathrm{fac}}+$ $B_{1}^{\text {pole }}$, and $B_{2}^{\text {DIR }}=B_{2}^{\text {fac }}+B_{2}^{\text {pole }}$ and their numerical values are presented in Table IV. Using the values in Table II, one can obtain $A^{\mathrm{DIR}}=A^{\text {fac }}+A^{\text {pole }}$ and $B^{\mathrm{DIR}}=B^{\text {fac }}+B^{\text {pole }}$. Summing up the contributions of the direct transition and that involving final state interaction we have $A$ and $B$ in the total amplitude $M_{a}$ and our theoretical results are presented in Table IV where the values in front of (or between) the parentheses are corresponding to those in Table III (a) (or (b)). The experimental results on $\Gamma\left(\Lambda_{c} \rightarrow \Sigma^{0} \pi^{+}\right)$and $\alpha$ are $(4.08 \pm 0.33) \times 10^{-14} \mathrm{GeV}[22]$ and $-0.73 \pm 0.17$ (stat) \pm 0.07 (syst) [26].

In this scenario, we have made a theoretical prediction on the up-down asymmetry $\alpha$ whose value resides within the error tolerance of the data and its sign is consistent with the experimental measurement. The predicted decay width of $\Gamma\left(\Lambda_{c} \rightarrow \Sigma \pi\right)$ is also closer to data than that made in Ref. [11]. Apparently final state interaction changes the naïve results of Ref. [11]. From Table II one can find that the signs of $A^{\text {pole }}$ and $B^{\text {pole }}$ are the same so the sign of $\alpha$ calculated in pole model is positive. As the final state interaction are taken into account the interference between the direct transition and the final state interaction would induce a conversion of the sign of the asymmetry parameter. Namely $A$ and $B$ (Table V) possess opposite signs, so the sign of $\alpha$ in is negative. It is noticed that, in this work, only the contribution from the decay $\Lambda_{c} \rightarrow \Lambda \rho$ as an intermediate state is accounted, and certainly in principle,

TABLE III. Theoretical results of $\Lambda_{c} \rightarrow \Lambda \rho^{+}$in pole model where $A_{1}^{\text {fac }}, A_{1}^{\text {pole }}, A_{2}^{\text {fac }}, A_{2}^{\text {pole }}, B_{1}^{\text {fac }}, B_{1}^{\text {pole }}, B_{2}^{\text {fac }}, B_{2}^{\text {pole }}$ are in units of $G_{F} V_{c s} V_{u d} \times 10^{-2} \mathrm{GeV}^{2}$ and $\Gamma$ is in units of $10^{-14} \mathrm{GeV}$.

\begin{tabular}{lcccccccccc}
\hline \hline$\Lambda_{c} \rightarrow \Lambda \rho^{+}$ & $A_{1}^{\text {fac }}$ & $A_{1}^{\text {pole }}$ & $A_{2}^{\text {fac }}$ & $A_{2}^{\text {pole }}$ & $B_{1}^{\text {fac }}$ & $B_{1}^{\text {pole }}$ & $B_{2}^{\text {fac }}$ & $B_{2}^{\text {pole }}$ & $\alpha$ & $\Gamma$ \\
\hline (a) & -8.64 & 0 & -0.71 & 0 & 13.33 & 0 & -2.99 & 0 & -0.30 & 14.13 \\
(b) & -8.28 & 0 & -0.68 & 0 & 12.77 & 0 & -2.87 & 0 & -0.30 & 12.97 \\
\hline \hline
\end{tabular}


TABLE IV. $A^{\mathrm{FSI}}$ and $B^{\mathrm{FSI}}$ in $M_{a}^{\mathrm{FSI}}$ (in units of $G_{F} V_{c s} V_{u d} \times$ $\left.10^{-2} \mathrm{GeV}^{2}\right)$.

\begin{tabular}{lcc}
\hline \hline$\Lambda_{1}$ & $A^{\text {FSI }}$ & $B^{\text {FSI }}$ \\
\hline $0.8 \mathrm{GeV}$ & $-3.26(-3.12)$ & $2.75(2.63)$ \\
$1.0 \mathrm{GeV}$ & $-4.68(-4.48)$ & $5.17(4.95)$ \\
\hline \hline
\end{tabular}

TABLE V. Theoretical total results of $\Lambda_{c} \rightarrow \Sigma^{0} \pi^{+}$where $A$ and $B$ are in units of $G_{F} V_{c s} V_{u d} \times 10^{-2} \mathrm{GeV}^{2}$ and $\Gamma$ is in units of $10^{-14} \mathrm{GeV}$.

\begin{tabular}{ccccc}
\hline \hline$\Lambda_{1}$ & $A$ & $B$ & $\alpha$ & $\Gamma$ \\
\hline $0.8 \mathrm{GeV}$ & $-1.02(-0.88)$ & $17.38(17.26)$ & $-0.29(-0.25)$ & $2.95(2.89)$ \\
$1.0 \mathrm{GeV}$ & $-2.44(-2.24)$ & $19.80(19.58)$ & $-0.64(-0.60)$ & $4.24(4.07)$ \\
\hline \hline
\end{tabular}

other decay portals of $\Lambda_{c}$ should also contribute to the same process via rescattering. A careful analysis indicates that those contributions are not as important as that of $\Lambda_{c} \rightarrow \Lambda \rho \rightarrow \Sigma \pi$, therefore, we ignore those coupled channels in this work.

\section{SUMMARY}

At the quark level the decay $\Lambda_{c} \rightarrow \Sigma \pi$ receives only the nonfactorizable $W$-exchange and internal $W$-emission contributions. Based on the valence quark model $[7,8]$ or the pole-model [9-11] these nonfactorizable diagrams were calculated while the resultant sign of the up-down asymmetry conflicts with data. Employing current algebra the authors $[13,14]$ obtained a negative up-down asymmetry as required by data. Generally current algebra can be applied to study the decays where a soft pseudoscalar meson is emitted. However the pion in $\Lambda_{c} \rightarrow \Sigma \pi$ is far from being soft so it is not natural to explain the data by using the current algebra. Following the approach in the references [18-21] we suggest that a final state interaction (or rescattering) in the decays of $\Lambda_{c}$ can contribute to the observed $\Lambda_{c} \rightarrow \Sigma \pi$. In terms of the effective interactions, coupling constants we calculate the contribution of the subprocess $\Lambda_{c} \rightarrow \Lambda \rho \rightarrow \Sigma \pi$ to the observed $\Lambda_{c} \rightarrow \Sigma \pi$. We notice (see the Table II), with the pole model, that the contribution to both $A^{\mathrm{DIR}}$ and $B^{\mathrm{DIR}}$ in Eq. (1) are positive while the contributions originating from the rescattering to $A^{\mathrm{FSI}}$ destructively interferes with that of $A^{\mathrm{DIR}}$ of the direct transition whereas $B^{\mathrm{FSI}}$ constructively interferes with $B^{\mathrm{DIR}}$; thus, as a consequence, the sign of the asymmetry parameter is reversed due to the destructive interference.

For our concrete calculations, generally considering, if there exists an absorptive part, it should dominate the rate. Thus we only calculate the absorptive part of the triangle (see the Feynman diagrams) where in the intermediate step $\Lambda$ and $\rho$ are on shell and we can factorize the two steps $\Lambda_{c} \rightarrow \Lambda \rho$ and $\Lambda \rho \rightarrow \Sigma \pi$. Including the contribution of the direct transition $\Lambda_{c} \rightarrow \Sigma \pi$ calculated using pole model and the subprocess $\Lambda_{c} \rightarrow \Lambda \rho \rightarrow \Sigma \pi$ which involves rescattering effects, we obtain a negative up-down asymmetry $\alpha$ and the resultant decay width of $\Gamma\left(\Lambda_{c} \rightarrow \Sigma \pi\right)$ is also closer to data than the original results of Ref. [11]. It is also noted that the rescattering of $\Lambda \rho$ to other products may reduce the observed rate of $\Lambda_{c} \rightarrow \Lambda \rho$. We predict the observed width of $\Lambda_{c} \rightarrow \Lambda \rho$ would be somehow smaller than the theoretically predicted value given in Table III.

In this paper we study the contribution of final state interaction to the transition $\Lambda_{c} \rightarrow \Sigma \pi$ and confirm that the final state interaction plays an important role in many hadronic transitions. In fact there still exist some discrepancies between theoretical estimations and data for other decays of $\Lambda_{c}$, and we hope the mechanism can also be applied to studying those "anomalies."

\section{ACKNOWLEDGMENTS}

This work is supported by the National Natural Science Foundation of China (NNSFC) under Contracts No. 12075167, No. 11375128, No. 11675082, and No. 11735010.
[1] N. Bilic, B. Guberina, and J. Trampetic, Nucl. Phys. B248, 261 (1984).

[2] G. Bellini, I. I. Y. Bigi, and P. J. Dornan, Phys. Rep. 289, 1 (1997).

[3] A. Zupanc et al. (Belle Collaboration), Phys. Rev. Lett. 113, 042002 (2014).

[4] M. Ablikim et al. (BESIII Collaboration), Phys. Rev. Lett. 116, 052001 (2016).

[5] P. Avery et al. (CLEO Collaboration), Phys. Rev. Lett. 65, 2842 (1990).

[6] H. Albrecht et al. (ARGUS Collaboration), Phys. Lett. B 269, 234 (1991).
[7] J. G. Korner and M. Kramer, Z. Phys. C 55, 659 (1992).

[8] M. A. Ivanov, J. G. Korner, V. E. Lyubovitskij, and A. G. Rusetsky, Phys. Rev. D 57, 5632 (1998).

[9] P. Zenczykowski, Phys. Rev. D 50, 5787 (1994).

[10] Q. P. Xu and A. N. Kamal, Phys. Rev. D 46, 270 (1992).

[11] H. Y. Cheng and B. Tseng, Phys. Rev. D 46, 1042 (1992).

[12] H. Y. Cheng and B. Tseng, Phys. Rev. D 48, 4188 (1993).

[13] K. K. Sharma and R. C. Verma, Eur. Phys. J. C 7, 217 (1999).

[14] H. Y. Cheng and B. Tseng, Phys. Rev. D 53, 1457 (1996).

[15] H. Y. Cheng, X. W. Kang, and F. Xu, Phys. Rev. D 97, 074028 (2018). 
[16] S. Weinberg, Phys. Rev. Lett. 18, 188 (1967).

[17] Y. S. Dai, D. S. Du, X. Q. Li, Z. T. Wei, and B. S. Zou, Phys. Rev. D 60, 014014 (1999).

[18] C. Meng and K. T. Chao, Phys. Rev. D 78, 034022 (2008).

[19] H. W. Ke, X. Q. Li, and X. Liu, Phys. Rev. D 82, 054030 (2010).

[20] X. H. Yuan, H. W. Ke, X. Liu, and X. Q. Li, Phys. Rev. D 87, 014019 (2013).

[21] H. Y. Cheng, C. K. Chua, and A. Soni, Phys. Rev. D 71, 014030 (2005).
[22] M. Tanabashi et al. (Particle Data Group) Phys. Rev. D 98, 030001 (2018).

[23] C. W. Shen, F. K. Guo, J. J. Xie, and B. S. Zou, Nucl. Phys. A954, 393 (2016).

[24] C. W. Shen, D. Rönchen, U. G. Meißner, and B. S. Zou, Chin. Phys. C 42, 023106 (2018).

[25] D. Ronchen, M. Doring, F. Huang, H. Haberzettl, J. Haidenbauer, C. Hanhart, S. Krewald, U. G. Meissner, and K. Nakayama, Eur. Phys. J. A 49, 44 (2013).

[26] M. Ablikim et al. (BESIII Collaboration) Phys. Rev. D 100, 072004 (2019). 